\title{
Pacific
}

Journal of

Mathematics

\section{TRACE-POSITIVE POLYNOMIALS}

IGOR KLEP 


\title{
TRACE-POSITIVE POLYNOMIALS
}

\author{
IGOR KLEP
}

\begin{abstract}
In this paper positivity of polynomials in free noncommuting variables in a dimension-dependent setting is considered. That is, the images of a polynomial under finite-dimensional representations of a fixed dimension are investigated. It is shown that unlike in the dimension-free case, every tracepositive polynomial is (after multiplication with a suitable denominator - a Hermitian square of a central polynomial) a sum of a positive semidefinite polynomial and commutators. Together with our previous results this yields the following Positivstellensatz: every trace-positive polynomial is modulo sums of commutators and polynomial identities a sum of Hermitian squares with weights and denominators. Understanding trace-positive polynomials is one of the approaches to Connes' embedding conjecture.
\end{abstract}

\section{Introduction}

Interest in positivity questions involving noncommutative polynomials has been recently revived by Helton's seminal paper [2002], in which he proved that a polynomial is a sum of squares if and only if its values in matrices of any size are positive semidefinite. Considering polynomials with positive trace, Klep and Schweighofer [2008, Theorem 1.6] observed that Connes' embedding conjecture [1976, Section V, pp. 105-107] on type $\mathrm{II}_{1}$ von Neumann algebras is equivalent to a problem of describing polynomials whose values at tuples of self-adjoint $d \times d$ matrices (of norm at most 1 ) have nonnegative trace for every $d \geq 1$. This result is the motivation for the present work. Here we investigate polynomials whose values at tuples of $d \times d$ matrices have nonnegative trace for a fixed $d \geq 1$. We show that such a polynomial is (after multiplication with a Hermitian square of a suitable central polynomial) a sum of commutators and of a polynomial whose values at tuples of $d \times d$ matrices are positive semidefinite. The latter were characterized in [Klep and Unger 2010], leading us to the following Positivstellensatz: every polynomial with nonnegative trace on $d \times d$ matrices is modulo sums of commutators and

Supported by the Slovenian Research Agency (project no. J1-3608 and program no. P1-0222). MSC2000: primary 16W10, 13J30; secondary 11E25, 16R50.

Keywords: free algebra, noncommutative polynomial, central simple algebra, (reduced) trace, polynomial identity, involution, central polynomial, quadratic form, free positivity. 
polynomial identities for $d \times d$ matrices a sum of Hermitian squares with weights and denominators. See Section 4 for a precise formulation.

The organization of this paper is as follows: Section 2 introduces the main notions and interprets them in full matrix algebras, Section 3 considers these notions for free algebras, while Section 4 presents our main results.

\section{Basic notions and a motivating example}

Let $R$ be an associative ring with 1 and involution $a \mapsto a^{*}$ (that is, $(a+b)^{*}=a^{*}+b^{*}$, $(a b)^{*}=b^{*} a^{*}$ and $a^{* *}=a$ for all $\left.a, b \in R\right)$. We denote by Sym $R:=\left\{a \in R \mid a=a^{*}\right\}$ its set of symmetric elements. Elements of the form $a^{*} a$ and $a b-b a(a, b \in$ $A)$ are called Hermitian squares and commutators, respectively. We introduce an equivalence relation (cyclic equivalence) on $R$ by declaring $a \stackrel{\text { cyc }}{\sim} b$ if and only if $a-b$ is a sum of commutators in $R$. For notational convenience we write

$$
\Sigma^{2} R:=\left\{\sum a_{i}^{*} a_{i} \mid a_{i} \in R\right\} \subseteq \operatorname{Sym} R, \quad \Theta^{2} R:=\left\{a \in R \mid \exists b \in \Sigma^{2} R: a \stackrel{\text { cyc }}{\sim} b\right\}
$$

for the sets of (finite) sums of Hermitian squares, and sums of Hermitian squares and commutators in $R$, respectively.

Throughout this paper $k$ will denote $\mathbb{R}$ or $\mathbb{C}$.

Matrices. For a concrete example of these notions, consider the ring $R=\mathrm{M}_{d}(k)$ of real or complex square matrices of a fixed size $d \geq 1$ endowed with the usual (complex conjugate) transposition of matrices, denoted here by $*$. Using $\succeq$ to denote the Löwner partial order (that is, $A \succeq B$ if and only if $A-B$ is positive semidefinite), it is easy to see that for $A \in \mathrm{M}_{d}(k)$, we have
(A) $A \succeq 0$ if and only if $A \in \Sigma^{2} \mathrm{M}_{d}(k)$;
(B) $\operatorname{tr}(A)=0$ if and only if $A \stackrel{\text { cyc }}{\sim} 0$ in $\mathrm{M}_{d}(k)$;
(C) $\operatorname{tr}(A) \geq 0$ if and only if $A \in \Theta^{2} \mathrm{M}_{d}(k)$.

Let us determine multiplication by which matrices respect these properties.

Lemma 2.1. Suppose $A \in \mathrm{M}_{d}(k)$ is such that for all $B \in \mathrm{M}_{d}(k)$,

$$
B \succeq 0 \quad \Rightarrow \quad A B \succeq 0 .
$$

Then $A=\lambda$ for some $\lambda \in \mathbb{R}_{\geq 0}$.

Proof. Using (1) with $B=1$, we obtain $A \succeq 0$. In particular, $A=A^{*}$. Again by (1), $A$ commutes with all positive semidefinite matrices, hence with all symmetric matrices, which are differences of two positive semidefinite matrices by

$$
B=\frac{1}{4}(B+1)^{2}-\frac{1}{4}(B-1)^{2} .
$$

So $A$ is scalar and the desired conclusion follows. 
Lemma 2.2. Suppose $A \in \mathrm{M}_{d}(k)$ is such that for all $B \in \mathrm{M}_{d}(k)$,

$$
\operatorname{tr}(B)=0 \Rightarrow \operatorname{tr}(A B)=0 .
$$

Then $A=\lambda$ for some $\lambda \in k$.

Proof. Write $A=\left[a_{i j}\right]_{i, j=1}^{d}$. Let $i \neq j$. Then $B=\lambda E_{i j}$ has zero trace for every $\lambda \in k$. (Here $E_{i j}$ denotes the $d \times d$ matrix unit with a one in position $(i, j)$ and zeros elsewhere.) By (2), this implies that $\lambda a_{i j}=\operatorname{tr}(A B)=0$. Since $\lambda \in k$ was arbitrary, $a_{i j}=0$.

Now let $B=\lambda\left(E_{i i}-E_{j j}\right)$. Clearly, $\operatorname{tr}(B)=0$ and hence

$$
\lambda\left(a_{i i}-a_{j j}\right)=\operatorname{tr}(A B)=0 .
$$

As before, this gives $a_{i i}=a_{j j}$.

Lemma 2.3. Suppose $A \in \mathrm{M}_{d}(k)$ is such that for all $B \in \mathrm{M}_{d}(k)$,

$$
\operatorname{tr}(B) \geq 0 \Rightarrow \operatorname{tr}(A B) \geq 0 .
$$

Then $A=\lambda$ for some $\lambda \in \mathbb{R}_{\geq 0}$.

Proof. By Lemma 2.2, $A$ is scalar. In addition to that, $a_{i i}=\operatorname{tr}\left(A E_{i i}\right) \geq 0$ by (3), showing that $A$ must be a nonnegative multiple of the identity.

Likewise we can characterize matrices that map positive semidefinite matrices into matrices with nonnegative trace:

Lemma 2.4. Suppose $A \in \mathrm{M}_{d}(k)$ is such that for all $B \in \mathrm{M}_{d}(k)$,

$$
B \succeq 0 \quad \Rightarrow \quad \operatorname{tr}(A B) \geq 0 .
$$

In the case $k=\mathbb{R}$, assume moreover that $A=A^{*}$. Then $A \succeq 0$.

Proof. This is just a restatement of the well-known self-duality of the cone of all positive semidefinite matrices. For $v \in k^{d}$, let $B=v v^{*} \succeq 0$. Then

$$
0 \leq \operatorname{tr}(A B)=\operatorname{tr}\left(A v v^{*}\right)=\operatorname{tr}\left(v^{*} A v\right)=\langle A v, v\rangle,
$$

showing $A$ is positive semidefinite.

Converses of Lemmas 2.1-2.4 hold as well.

\section{Positivity in free algebras}

Words and polynomials. Fix $n \in \mathbb{N}$. Let

$$
\underline{X}:=\left(X_{1}, \ldots, X_{n}\right) \quad \text { and } \quad \underline{X}^{*}:=\left(X_{1}^{*}, \ldots, X_{n}^{*}\right)
$$

denote tuples of $n$ distinct variables (or letters). By $\left\langle\underline{X}, \underline{X}^{*}\right\rangle$ we denote the free monoid on $\left\{\underline{X}, \underline{X}^{*}\right\}$ (consisting of words in $\underline{X}, \underline{X}^{*}$ ) and let $k\left\langle\underline{X}, \underline{X}^{*}\right\rangle$ be the semigroup algebra of $\left\langle\underline{X}, \underline{X}^{*}\right\rangle$ over $k$ (consisting of polynomials in noncommuting 
variables $\underline{X}$ and $\underline{X}^{*}$ with coefficients in $k$ ). We endow $k\left\langle\underline{X}, \underline{X}^{*}\right\rangle$ with the involution $p \mapsto p^{*}$ mapping $X_{j} \mapsto X_{j}^{*}$ and extending complex conjugation on $k$. Thus $k\left\langle\underline{X}, \underline{X}^{*}\right\rangle$ is the free $*$-algebra on $\underline{X}$ over $k$.

Cyclic equivalence. It is well known and easy to see that trace-zero matrices are sums of commutators, that is, cyclically equivalent to 0 . Cyclic equivalence can also be easily tested in $k\left\langle\underline{X}, \underline{X}^{*}\right\rangle$ :

(a) For $v, w \in\left\langle\underline{X}, \underline{X}^{*}\right\rangle$, we have $v \stackrel{\text { cyc }}{\sim} w$ if and only if there are $v_{1}, v_{2} \in\left\langle\underline{X}, \underline{X}^{*}\right\rangle$ such that $v=v_{1} v_{2}$ and $w=v_{2} v_{1}$. That is, $v \stackrel{\text { cyc }}{\sim} w$ if and only if $w$ is a cyclic permutation of $v$.

(b) Polynomials

$$
f=\sum_{w \in\left\langle\underline{X}, \underline{X}^{*}\right\rangle} a_{w} w \quad \text { and } \quad g=\sum_{w \in\left\langle\underline{X}, \underline{X}^{*}\right\rangle} b_{w} w \quad \text { for } a_{w}, b_{w} \in k
$$

are cyclically equivalent if and only if for each $v \in\left\langle\underline{X}, \underline{X}^{*}\right\rangle$,

$$
\sum_{\substack{w \in\left\langle\underline{X}, \underline{X^{*}}\right\rangle \\ \text { cyc } \\ w \sim v}} a_{w}=\sum_{\substack{w \in\left\langle\underline{X}, \underline{X^{*}}\right\rangle \\ w \sim v \\ w \sim v}} b_{w} .
$$

Evaluations and representations. Let $d \in \mathbb{N}$. An $n$-tuple of matrices $\underline{A} \in\left(\mathrm{M}_{d}(k)\right)^{n}$ gives rise to a $*$-representation

$$
\mathrm{ev}_{\underline{A}}: k\left\langle\underline{X}, \underline{X}^{*}\right\rangle \rightarrow \mathrm{M}_{d}(k), \quad p \mapsto p\left(\underline{A}, \underline{A}^{*}\right) .
$$

We are interested in the values of a fixed element $f \in k\left\langle\underline{X}, \underline{X}^{*}\right\rangle$ under all these *-representations. If the size $d$ of the matrices $A_{i}$ is free, we talk about dimensionfree properties; otherwise we call them dimension-dependent. We are mostly interested in the latter, but briefly review the former for the sake of completeness.

Dimension-freeness. Free analogs of properties (A) and (B) have been established, while a free version of $(\mathrm{C})$ is closely related to an important open problem on operator algebras due to Connes; see below for further details.

Let $f \in \operatorname{Sym} k\left\langle\underline{X}, \underline{X}^{*}\right\rangle$.

(A) $)^{\mathrm{fr}} f\left(\underline{A}, \underline{A}^{*}\right) \geq 0$ for all $d \in \mathbb{N}$ and all $\underline{A} \in \mathrm{M}_{d}(k)^{n}$ if and only if $f \in \Sigma^{2} k\left\langle\underline{X}, \underline{X}^{*}\right\rangle$;

$(\mathrm{B})^{\mathrm{fr}} \operatorname{tr}\left(f\left(\underline{A}, \underline{A}^{*}\right)\right)=0$ for all $d \in \mathbb{N}$ and all $\underline{A} \in \mathrm{M}_{d}(k)^{n}$ if and only if $f \stackrel{\text { cyc }}{\sim} 0$ in $k\left\langle\underline{X}, \underline{X}^{*}\right\rangle$.

Part (A) ${ }^{\text {fr }}$ is due to Helton [2002] (see also [McCullough 2001; McCullough and Putinar 2005]), and (B) ${ }^{\mathrm{fr}}$ is Theorem 2.1 of [Klep and Schweighofer 2008]. (This reference will henceforth be abbreviated as [KS 2008].) See also [Collins and Dykema 2008, Lemma 2.9] for a proof inspired by free probability. For a recent 
study of trace-positive polynomials in a dimension-free setting see also [Netzer and Thom 2010].

The obvious extension of (C) fails: there are $f \in \operatorname{Sym} k\left\langle\underline{X}, \underline{X}^{*}\right\rangle$ with positive trace everywhere, but still not cyclically equivalent to a sum of Hermitian squares. The following is a variant of the noncommutative Motzkin polynomial from Example 4.4 of [KS 2008] given in free (nonsymmetric) variables.

Example 3.1. Let $X$ denote a single free variable and set

$$
\begin{aligned}
& M_{0}:= \\
& 3 X^{4}-3\left(X X^{*}\right)^{2}-4 X^{5} X^{*}-2 X^{3} X^{* 3}+2 X^{2} X^{*} X X^{* 2}+2 X^{2} X^{* 2} X X^{*}+2\left(X X^{*}\right)^{3} .
\end{aligned}
$$

Then the noncommutative Motzkin polynomial is

$$
M:=1+M_{0}+M_{0}^{*} \in \operatorname{Sym} k\left\langle X, X^{*}\right\rangle .
$$

It is trace-nonnegative everywhere since

$$
M^{\prime}:=Y Z^{4} Y+Z Y^{4} Z-3 Y Z^{2} Y+1 \stackrel{\text { cyc }}{\sim} M\left(\frac{Y+\dot{\mathrm{i}} Z}{2}, \frac{Y-\dot{\mathrm{i}} Z}{2}\right) \in k\langle Y, Z\rangle
$$

is trace-nonnegative on symmetric matrices; see Example 4.4 of [KS 2008]. Alternatively, $M\left(X^{3},\left(X^{*}\right)^{3}\right) \in \Theta^{2} k\left\langle X, X^{*}\right\rangle$. On the other hand, $M \notin \Theta^{2} k\left\langle X, X^{*}\right\rangle$. (Some of these computations were done with the aid of the computer algebra systems NCSOStools [Cafuta et al. 2010] and NCAlgebra [Helton et al. 2010].)

Connes' embedding conjecture [1976, Section V, pp. 105-107] states that every separable $\mathrm{II}_{1}$-factor is embeddable in an ultrapower of the hyperfinite $\mathrm{II}_{1}$-factor. Understanding trace-positive polynomials in the dimension-free setting is the key to this problem, because it is equivalent, by Theorem 1.6 of [KS 2008], to Conjecture 1.5 of the same reference, which we repeat here for convenience:

Conjecture 3.2 (algebraic version of Connes' conjecture). For $f \in \operatorname{Sym} k\left\langle\underline{X}, \underline{X}^{*}\right\rangle$ the following are equivalent:

(i) $\operatorname{tr}\left(f\left(\underline{A}, \underline{A}^{*}\right)\right) \geq 0$ for all $d \in \mathbb{N}$ and all tuples of contractions $\underline{A} \in \mathrm{M}_{d}(k)^{n}$;

(ii) for every $\varepsilon \in \mathbb{R}_{>0}, f+\varepsilon$ is cyclically equivalent to an element of the form

$$
\sum_{j} s_{j}^{*} s_{j}+\sum_{i, j} p_{i j}^{*}\left(1-X_{i}^{*} X_{i}\right) p_{i j}
$$

where $s_{j}, p_{i j} \in k\left\langle\underline{X}, \underline{X}^{*}\right\rangle$.

In the sequel we indicate an approach to this problem "from below". That is, we abandon the dimension-free setting and solve a Hilbert 17-type problem characterizing polynomials with nonnegative trace in a dimension-dependent setting. It is our belief that this might constitute an important step towards (a positive or negative resolution of) Connes' embedding conjecture. 


\section{Dimension-dependent positivity}

The properties (A) and (B) for free algebras in a dimension-dependent setting are well understood due to our previous work. Roughly speaking, a trace-zero polynomial is cyclically equivalent to a polynomial identity [Brešar and Klep 2009, Section 4], and a positive semidefinite polynomial is a sum of Hermitian squares with denominators and weights [Klep and Unger 2010, Section 5]. In this section property $(\mathrm{C})$ is explored and we present our main result, a Positivstellensatz characterizing polynomials with nonnegative trace on all tuples of $d \times d$ matrices for fixed $d$. This is done in Section 4C. Before that we recall generic matrices and universal division algebras with involution in Section 4A and take a look at polynomial preservers of the various notions of positivity in Section 4B.

4A. Generic matrices and universal division algebras. We assume the reader is familiar with the theory of polynomial identities as presented, e.g., in [Procesi 1973; Rowen 1980]. We review the notion of generic matrices and universal division algebras with involution and refer the reader to [Procesi 1976; Procesi and Schacher 1976] for details.

Let $\zeta:=\left(\zeta_{i j}^{(\ell)} \mid 1 \leq i, j \leq d, 1 \leq \ell \leq n\right)$ and $\bar{\zeta}:=\left(\bar{\zeta}_{i j}^{(\ell)} \mid 1 \leq i, j \leq d, 1 \leq \ell \leq n\right)$ denote commuting variables. To keep the notation uniform, let

$$
\zeta:= \begin{cases}\zeta & \text { if } k=\mathbb{R} \\ (\zeta, \bar{\zeta}) & \text { if } k=\mathbb{C}\end{cases}
$$

Form the polynomial $*$-algebra $k[\underline{\zeta}]$ that endowed with the involution that extends complex conjugation on $k$ and fixes $\zeta_{i j}^{(\ell)}$ pointwise (if $k=\mathbb{R}$ ) or sends $\zeta_{i j}^{(\ell)}$ to $\bar{\zeta}_{i j}^{(\ell)}$ (if $k=\mathbb{C}$ ).

Consider the $d \times d$ matrices

$$
Y_{\ell}:=\left[\zeta_{i j}^{(\ell)}\right]_{1 \leq i, j \leq d} \in \mathrm{M}_{d}(k[\underline{\zeta}]) \quad \text { for } \ell \in \mathbb{N} .
$$

Each $Y_{\ell}$ is called a generic matrix. The (unital) $k$-subalgebra of $\mathrm{M}_{d}(k[\zeta])$ generated by the $Y_{\ell}$ and their (complex conjugate) transposes is the ring of generic matrices with involution $\mathrm{GM}_{d}(k)$. Equivalently,

$$
\mathrm{GM}_{d}(k) \cong k\left\langle\underline{X}, \underline{X}^{*}\right\rangle / \mathfrak{t}_{d},
$$

where $\mathfrak{t}_{d} \subseteq k\left\langle\underline{X}, \underline{X}^{*}\right\rangle$ is the T-ideal of polynomial identities for $d \times d$ matrices.

For $d \geq 2$, the ring $\mathrm{GM}_{d}(k)$ is a prime PI algebra (see [Procesi and Schacher 1976, Section II]). Hence its central localization is a central simple algebra $\mathrm{UD}_{d}(k)$ with involution, which we call (by an abuse of notation) the universal division algebra. Relating these notions to $*$-representations of the free $*$-algebra is the following commutative diagram: for $d \in \mathbb{N}$ and $\underline{A} \in \mathrm{M}_{d}(k)^{n}$, let $R_{\underline{A}}$ denote all the 
elements of $\mathrm{UD}_{d}(k)$ that are regular at $\underline{A}$. Then:

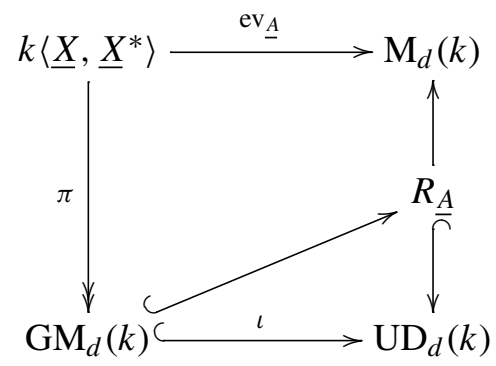

For a more geometric viewpoint of the ring of generic matrices and the universal division algebra we refer the reader to [Procesi 1976; Saltman 1999]. The standard textbook on central simple algebras with involution is [Knus et al. 1998].

4B. Polynomial preservers. In this subsection we present versions of Lemmas 2.1-2.4 in the context of free $*$-algebras. To avoid trivialities, we assume throughout that $d \geq 2$.

Lemma 4.1. Suppose $f \in k\left\langle\underline{X}, \underline{X}^{*}\right\rangle$ is such that for all $g \in k\left\langle\underline{X}, \underline{X}^{*}\right\rangle$,

$$
g \succeq 0 \text { on } d \times d \text { matrices } \quad \Rightarrow \quad f g \succeq 0 \text { on } d \times d \text { matrices. }
$$

Then $f$ is a central polynomial positive semidefinite on $d \times d$ matrices.

Proof. Using (7) with $g=1$, we see $f$ is positive semidefinite on $d \times d$ matrices. Thus there is no harm in assuming $f=f^{*}$.

Again by (7), $f g-g f$ vanishes on all $d \times d$ matrices for all polynomials $g$ of the form $g=h^{*} h$. That is, $[f, g]$ is a polynomial identity of $d \times d$ matrices. Now the same holds true for all symmetric $g$, since

$$
2[f, g]+\left[f, g^{2}\right]=\left[f,(1+g)^{2}\right]
$$

is then a polynomial identity. Hence $f$ commutes (modulo the T-ideal of identities) with all symmetric polynomials.

Every element of $\mathrm{UD}_{d}(k)$ can be represented as $r s^{-1}$ for some $r, s \in \mathrm{GM}_{d}(k)$ with $s=s^{*} \in Z\left(\mathrm{GM}_{d}(k)\right)$. Such an element is symmetric if and only if $r=r^{*}$. So $\pi(f)$ commutes with all symmetric elements of $\operatorname{UD}_{d}(k)$. By Dieudonné's theorem [1952, Lemma 1], the latter generate $\operatorname{UD}_{d}(k)$. Hence $\pi(f) \in Z\left(\operatorname{UD}_{d}(k)\right)$ and $f$ is indeed a central polynomial.

(Note: once we have established that $f$ commutes with all symmetric polynomials, an easier argument is available if $k=\mathbb{C}$. In this case one immediately obtains that $f$ also commutes with all skew symmetric polynomials as these are all of the form in for symmetric $g$.) 
Lemma 4.2. Suppose $f \in k\left\langle\underline{X}, \underline{X}^{*}\right\rangle$ is such that for all $g \in k\left\langle\underline{X}, \underline{X}^{*}\right\rangle$,

$$
\operatorname{tr}(g)=0 \text { on } d \times d \text { matrices } \Rightarrow \operatorname{tr}(f g)=0 \text { on } d \times d \text { matrices. }
$$

Then $f$ is a central polynomial.

Proof. Let $g=\left[h_{1}, h_{2}\right]$ for some $h_{i} \in k\left\langle\underline{X}, \underline{X}^{*}\right\rangle$. Then

$$
f g=f\left[h_{1}, h_{2}\right]=\left[f, h_{1} h_{2}\right]+\left[h_{1}, f h_{2}\right]+h_{1}\left[h_{2}, f\right] .
$$

Since $\operatorname{tr}(g)=0$ on all $d \times d$ matrices, this implies $\operatorname{tr}\left(h_{1}\left[h_{2}, f\right]\right)=0$ on $d \times d$ matrices. Fix $h_{2}$ and denote $r:=\left[h_{2}, f\right]$. Then $r$ satisfies

$$
\operatorname{tr}(p r)=0 \text { on } d \times d \text { matrices }
$$

for all $p \in k\left\langle\underline{X}, \underline{X}^{*}\right\rangle$. Taking $p=-r^{*}$ leads to $-\operatorname{tr}\left(r^{*} r\right)=0$, and hence $r=0$ on all $d \times d$ matrices. That is, $r$ is an identity of $d \times d$ matrices. As $r=\left[h_{2}, f\right]$ and $h_{2}$ was arbitrary, this implies $f$ is a central polynomial.

Lemma 4.3. Suppose $f \in k\left\langle\underline{X}, \underline{X}^{*}\right\rangle$ is such that for all $g \in k\left\langle\underline{X}, \underline{X}^{*}\right\rangle$,

$$
\operatorname{tr}(g) \geq 0 \text { on } d \times d \text { matrices } \Rightarrow \operatorname{tr}(f g) \geq 0 \text { on } d \times d \text { matrices. }
$$

Then $f$ is a central polynomial positive semidefinite on $d \times d$ matrices.

Proof. If $\operatorname{tr}(g)=0$, then by (10), $\operatorname{tr}(f g) \geq 0$ and $\operatorname{tr}(-f g) \geq 0$ on $d \times d$ matrices. That is, $\operatorname{tr}(f g)=0$. Now by Lemma 4.2, $f$ is a central polynomial.

Applying (10) with $g=1$ yields $f\left(\underline{A}, \underline{A}^{*}\right)=\operatorname{tr}\left(f\left(\underline{A}, \underline{A}^{*}\right)\right) \geq 0$ for all $\underline{A} \in \mathrm{M}_{d}(k)^{n}$, showing $f$ is positive semidefinite on $d \times d$ matrices.

Likewise we can characterize polynomials that map positive semidefinite polynomials into trace-nonnegative ones. At the same time this indicates how to build examples of trace-nonnegative polynomials. As we shall see in the next subsection, the procedure is essentially exhaustive.

Lemma 4.4. Suppose $f \in \operatorname{Sym} k\left\langle\underline{X}, \underline{X}^{*}\right\rangle$ is such that for all $g \in k\left\langle\underline{X}, \underline{X}^{*}\right\rangle$,

$$
g \succeq 0 \text { on } d \times d \text { matrices } \Rightarrow \operatorname{tr}(f g) \geq 0 \text { on } d \times d \text { matrices. }
$$

Then $f$ is positive semidefinite on $d \times d$ matrices.

Proof. Assume $f$ is not positive semidefinite on $d \times d$ matrices. Then there exists an $n$-tuple $\underline{A}=\left(A_{1}, \ldots, A_{n}\right) \in \mathrm{M}_{d}(k)^{n}$ with

$$
f\left(\underline{A}, \underline{A}^{*}\right) \nsucceq 0 .
$$

Let $\mathscr{A} \subseteq \mathrm{M}_{d}(k)$ denote the $*$-subalgebra generated by the $A_{1}, \ldots, A_{n}$. Since the Hermitian square of a nonzero matrix is not nilpotent, $\mathscr{A}$ is semisimple. By the Artin-Wedderburn theorem, $\mathscr{A}$ is $*$-isomorphic to a direct sum of full matrix algebras. We distinguish two cases. 
CASE 1: If $k=\mathbb{C}$, there is a $*$-isomorphism

$$
\mathscr{A} \cong \bigoplus_{j=1}^{s} \mathrm{M}_{d_{j}}(\mathbb{C})
$$

for some $d_{j} \in \mathbb{C}$, and $\sum_{j} d_{j} \leq d$. This induces a block diagonalization

$$
A_{j}=\left[\begin{array}{lll}
A_{j, 1} & & \\
& \ddots & \\
& & A_{j, s}
\end{array}\right], \quad \text { with } A_{j, k} \in \mathrm{M}_{d_{k}}(\mathbb{C}) .
$$

By (12), there is a $j$ such that $\underline{A}_{(j)}=\left(A_{1, j}, \ldots, A_{n, j}\right) \in \mathrm{M}_{d_{j}}(\mathbb{C})^{n}$ satisfies

$$
f\left(\underline{A}_{(j)}, \underline{A}_{(j)}^{*}\right) \nsucceq 0 .
$$

Choose $u \in \mathbb{C}^{d_{j}}$ with

$$
\left\langle f\left(\underline{A}_{(j)}, \underline{A}_{(j)}^{*}\right) u, u\right\rangle<0 .
$$

There is a $B \in \mathrm{M}_{d_{j}}(\mathbb{C})$ with $B e_{i, d_{j}}=u$ for all $i=1, \ldots, d_{j}$. (Here $e_{i, d_{j}}$ are the standard basis vectors for $\mathbb{C}^{d_{j}}$.) By the construction of $\mathscr{A}$ and (13), there is an $h \in \mathbb{C}\left\langle\underline{X}, \underline{X}^{*}\right\rangle$ with $h\left(\underline{A}_{(j)}, \underline{A}_{(j)}^{*}\right)=B$. Let $g=h h^{*}$. Then

$$
\begin{aligned}
\operatorname{tr}\left((f g)\left(\underline{A}_{(j)}, \underline{A}_{(j)}^{*}\right)\right) & =\operatorname{tr}\left(\left(h^{*} f h\right)\left(\underline{A}_{(j)}, \underline{A}_{(j)}^{*}\right)\right) \\
& =\sum_{i=1}^{d_{j}}\left\langle h^{*}\left(\underline{A}_{(j)}, \underline{A}_{(j)}^{*}\right) f\left(\underline{A}_{(j)}, \underline{A}_{(j)}^{*}\right) h\left(\underline{A}_{(j)}, \underline{A}_{(j)}^{*}\right) e_{i, d_{j}}, e_{i, d_{j}}\right\rangle \\
& =\sum_{i=1}^{d_{j}}\left\langle f\left(\underline{A}_{(j)}, \underline{A}_{(j)}^{*}\right) B e_{i, d_{j}}, B e_{i, d_{j}}\right\rangle \\
& =\sum_{i=1}^{d_{j}}\left\langle f\left(\underline{A}_{(j)}, \underline{A}_{(j)}^{*}\right) u, u\right\rangle<0 .
\end{aligned}
$$

As this contradicts our assumption (11), we conclude that $f \succeq 0$ on $d \times d$ matrices. CASE 2: If $k=\mathbb{R}$, the reasoning is the same with a minor technical modification. Let

$$
\mathscr{A} \cong \bigoplus_{j=1}^{s} \mathrm{M}_{d_{j}}(\mathbb{R}) \oplus \bigoplus_{k=1}^{r} \mathrm{M}_{e_{k}}(\mathbb{C}) \oplus \bigoplus_{\ell=1}^{p} \mathrm{M}_{f_{\ell}}(\mathbb{M})
$$

for some $d_{j}, e_{k}, f_{\ell} \in \mathbb{N}$.

If there is a tuple $\underline{A} \in \mathrm{M}_{d_{j}}(\mathbb{R})^{n}$ with $f\left(\underline{A}, \underline{A}^{*}\right) \nsucceq 0$, we proceed as in Case 1. If there is an $\underline{A} \in \mathrm{M}_{e_{k}}(\mathbb{C})^{n}$ with $0 \npreceq f\left(\underline{A}, \underline{A}^{*}\right) \in \mathrm{M}_{e_{k}}(\mathbb{C})$, we proceed as follows. Let $V$ be the invariant subspace of $\mathbb{R}^{d}$ corresponding to the action of $\mathrm{M}_{e_{k}}(\mathbb{C})$. There is a $u \in V$ with $\left\langle f\left(\underline{A}, \underline{A}^{*}\right) u, u\right\rangle<0$. Pick a basis $\left\{v_{1}, \ldots, v_{e_{k}}\right\}$ of $V$ over $\mathbb{C}$, and let 
$B \in \mathrm{M}_{e_{k}}(\mathbb{C})$ satisfy $B v_{j}=u$ for all $j$. Choose $h \in \mathbb{R}\left\langle\underline{X}, \underline{X}^{*}\right\rangle$ with $h\left(\underline{A}, \underline{A}^{*}\right)=B$ and $g=h h^{*}$. Then the complex trace $z$ of $(f g)\left(\underline{A}, \underline{A}^{*}\right)$ is negative by the same computation as in (15). Hence the real trace satisfies

$$
\operatorname{tr}\left((f g)\left(\underline{A}, \underline{A}^{*}\right)\right)=\frac{z+\bar{z}}{2}<0 .
$$

The remaining case of quaternion matrices is dealt with similarly. We leave this as an exercise for the reader.

It is clear that converses of Lemmas 4.1-4.4 hold true. Also, with the exception of (11), which is satisfied when $f$ is a sum of Hermitian squares, there are no nonconstant dimension-free polynomial preservers.

4C. The dimension-dependent tracial Positivstellensatz. Our main tool for describing trace-nonnegative polynomials is the following proposition deduced from the properties of the reduced trace [Knus et al. 1998, Section 1] on $\mathrm{UD}_{d}(k)$.

Proposition 4.5. For every $f \in k\left\langle\underline{X}, \underline{X}^{*}\right\rangle$ and $d \in \mathbb{N}$ there exists a nonvanishing central polynomial for $d \times d$ matrices, denoted by $c \in k\left\langle\underline{X}, \underline{X}^{*}\right\rangle$, such that $c f$ is cyclically equivalent to a central polynomial. That is,

$$
c f \stackrel{\text { cyc }}{\sim} c^{\prime}
$$

for some central polynomial c'.

Proof. Consider $F:=\iota(\pi(f)) \in \mathrm{UD}_{d}(k)$. So $\operatorname{Trd}(F) \in Z\left(\mathrm{UD}_{d}(k)\right)$, and there is a nonvanishing central polynomial $c_{0} \in k\left\langle\underline{X}, \underline{X}^{*}\right\rangle$ and a central polynomial $c_{0}^{\prime}$ with

$$
\operatorname{Trd}(F)=\pi\left(c_{0}^{\prime}\right) \pi\left(c_{0}\right)^{-1} .
$$

Since $\operatorname{Trd}$ is $Z\left(\mathrm{UD}_{d}(k)\right)$-linear, this yields $\operatorname{Trd}\left(\pi\left(c_{0} f-c_{0}^{\prime}\right)\right)=0$. By [Amitsur and Rowen 1994, Theorem 2.4], $\pi\left(c_{0} f-c_{0}^{\prime}\right) \stackrel{\text { cyc }}{\sim} 0$ in $\operatorname{UD}_{d}(k)$. Clearing denominators shows

$$
\pi\left(c f-c^{\prime \prime}\right) \stackrel{\text { cyc }}{\sim} 0
$$

in $\mathrm{GM}_{d}(k)$ for a nonvanishing central polynomial $c$ and a central polynomial $c^{\prime \prime}$. Lifting (19) to $k\left\langle\underline{X}, \underline{X}^{*}\right\rangle$ gives the desired conclusion: $c f \stackrel{\text { cyc }}{\sim} c^{\prime}$.

Remark 4.6. Instead of the Amitsur-Rowen result used in this proof, we can apply the tracial Nullstellensatz [Brešar and Klep 2009, Theorem 5.2]: once we have established that $\operatorname{Trd}\left(\pi\left(c_{o} f-c_{0}^{\prime}\right)\right)=0$, by clearing denominators we obtain $\operatorname{tr}\left(\pi\left(c_{0} c^{\prime \prime} f-c_{0}^{\prime} c^{\prime \prime}\right)\right)=0$ for some nonvanishing central polynomial $c^{\prime \prime}$. Hence $\pi\left(c_{0} c^{\prime \prime} f-c^{\prime} c^{\prime \prime}\right) \stackrel{\text { cyc }}{\sim} 0$ in $\mathrm{GM}_{d}(k)$ by [Brešar and Klep 2009, Theorem 5.2]. As before, lifting this relation to $k\left\langle\underline{X}, \underline{X}^{*}\right\rangle$ yields the desired conclusion. 
We are now ready to give our main results characterizing trace-nonnegative polynomials.

Theorem 4.7. Let $k \in\{\mathbb{R}, \mathbb{C}\}$ and suppose $f \in \operatorname{Sym} k\left\langle\underline{X}, \underline{X}^{*}\right\rangle$ satisfies

$$
\operatorname{tr}\left(f\left(\underline{A}, \underline{A}^{*}\right)\right) \geq 0
$$

for all $\underline{A} \in \mathrm{M}_{d}(k)^{n}$. Then there is a nonvanishing central polynomial for $d \times d$ matrices, denoted by $c \in k\left\langle\underline{X}, \underline{X}^{*}\right\rangle$, such that $c f c^{*}$ is cyclically equivalent to a polynomial $g \in k\left\langle\underline{X}, \underline{X}^{*}\right\rangle$ that is positive semidefinite on $d \times d$ matrices:

$$
c f c \stackrel{\text { cyc }}{\sim} g \text { and } g \succeq 0 \text { on } d \times d \text { matrices. }
$$

Proof. This is a consequence of Proposition 4.5. Indeed, there is a nonvanishing central polynomial $c$ with

$$
c f \stackrel{\text { cyc }}{\sim} c^{\prime}
$$

for a central polynomial $c^{\prime}$. Multiplying (22) with $c^{*}$ (from the right) shows

$$
c f c^{*} \stackrel{\text { cyc }}{\sim} c^{\prime} c^{*} \text {. }
$$

For any $\underline{A} \in \mathrm{M}_{d}(k)^{n}$,

$$
\begin{aligned}
0 & \leq \operatorname{tr}\left(c\left(\underline{A}, \underline{A}^{*}\right) f\left(\underline{A}, \underline{A}^{*}\right) c\left(\underline{A}, \underline{A}^{*}\right)^{*}\right)=\operatorname{tr}\left(c^{\prime}\left(\underline{A}, \underline{A}^{*}\right) c\left(\underline{A}, \underline{A}^{*}\right)^{*}\right) \\
& =\operatorname{tr}\left(\left(c^{\prime} c^{*}\right)\left(\underline{A}, \underline{A}^{*}\right)\right)=\left(c^{\prime} c^{*}\right)\left(\underline{A}, \underline{A}^{*}\right) .
\end{aligned}
$$

So $g:=c^{\prime} c^{*}$ is a (central) polynomial positive semidefinite on $d \times d$ matrices satisfying

$$
c f c \stackrel{\text { cyc }}{\sim} g .
$$

Remark 4.8. The proof shows that $g$ in Theorem 4.7 can actually be taken to be a central polynomial.

Combining Theorem 4.7 with the dimension-dependent Positivstellensatz for positive semidefinite polynomials ([Procesi and Schacher 1976, Theorem 5.4] or [Klep and Unger 2010, Theorem 5.4]) yields:

Corollary 4.9. Choose $\alpha_{1}, \ldots, \alpha_{m} \in k\left\langle\underline{X}, \underline{X}^{*}\right\rangle$ whose images in $\mathrm{GM}_{d}(k)$ form a diagonalization of the quadratic form $\operatorname{Trd}\left(x^{*} x\right)$ on $\mathrm{UD}_{d}(k)$. For $f \in \operatorname{Sym} k\left\langle\underline{X}, \underline{X}^{*}\right\rangle$, the following are equivalent:

(i) $\operatorname{tr}\left(f\left(\underline{A}, \underline{A}^{*}\right)\right) \geq 0$ for every $\underline{A} \in \mathrm{M}_{d}(k)^{n}$.

(ii) There exists a nonvanishing central polynomial $c \in k\left\langle\underline{X}, \underline{X}^{*}\right\rangle$, a polynomial identity $h \in k\left\langle\underline{X}, \underline{X}^{*}\right\rangle$ for $d \times d$ matrices, and $p_{i, \varepsilon} \in k\left\langle\underline{X}, \underline{X}^{*}\right\rangle$ with

$$
c f c^{*} \stackrel{\text { cyc }}{\sim} h+\sum_{\varepsilon \in\{0,1\}^{m}} \underline{\alpha}^{\varepsilon} \sum_{i} p_{i, \varepsilon}^{*} p_{i, \varepsilon} .
$$


Remark 4.10. For experts we mention that, by applying the reduced trace, we can reformulate (25) as

$$
c f c \stackrel{\text { cyc }}{\sim} h+t,
$$

where $c$ and $h$ are as above, and $t$ belongs to the preordering in $Z\left(\operatorname{UD}_{d}(k)\right)$ generated by the $\alpha_{j}$.

If $d=2$, the weights $\alpha_{j}$ are superfluous since the reduced trace of a Hermitian square is a sum of Hermitian squares in this case (see [Procesi and Schacher 1976, p. 405] or [Klep and Unger 2010, Section 4]), and Corollary 4.9 simplifies as follows:

Corollary 4.11. For $f \in \operatorname{Sym} k\left\langle\underline{X}, \underline{X}^{*}\right\rangle$, the following are equivalent:

(i) $\operatorname{tr}\left(f\left(\underline{A}, \underline{A}^{*}\right)\right) \geq 0$ for every $\underline{A} \in \mathrm{M}_{2}(k)^{n}$.

(ii) There exists a nonvanishing central polynomial $c \in k\left\langle\underline{X}, \underline{X}^{*}\right\rangle$, and a polynomial identity $h \in k\left\langle\underline{X}, \underline{X}^{*}\right\rangle$ for $2 \times 2$ matrices, such that

$$
c f c^{*} \in h+\Theta^{2} k\left\langle\underline{X}, \underline{X}^{*}\right\rangle .
$$

Example 4.12. We finish this presentation with an example showing denominators are necessary for these results to hold. First, the Motzkin polynomial $M$ from Example 3.1 is not cyclically equivalent to a sum of Hermitian squares modulo a T-ideal of identities. Indeed, suppose that

$$
M \stackrel{\text { cyc }}{\sim} h+\sum g_{j}^{*} g_{j}
$$

for some $g_{j} \in k\left\langle\underline{X}, \underline{X}^{*}\right\rangle$ and a polynomial identity $h \in k\left\langle\underline{X}, \underline{X}^{*}\right\rangle$ for $d \times d$ matrices $(d \geq 2)$. Then

$$
M_{\mathrm{cc}}=\operatorname{tr}\left(M\left(\left[\begin{array}{rr}
Y / 2 & Z / 2 \\
-Z / 2 & Y / 2
\end{array}\right]\right)\right)=\sum \operatorname{tr}\left(\left(g_{j}^{*} g_{j}\right)\left(\left[\begin{array}{rr}
Y / 2 & Z / 2 \\
-Z / 2 & Y / 2
\end{array}\right]\right)\right),
$$

where $M_{\mathrm{cc}} \in \mathbb{R}[Y, Z]$ denotes the commutative collapse $Y^{4} Z^{2}+Y^{2} Z^{4}-3 Y^{2} Z^{2}+1$ of the noncommutative variant $M^{\prime}$ of the Motzkin polynomial (in symmetric variables). Since $M_{\mathrm{cc}}$ is not a sum of squares in $\mathbb{R}[Y, Z]$, and the trace of a Hermitian square is a sum of squares, $M$ does not satisfy a relation of the form (28). Hence a denominator is needed in Corollaries 4.9 and 4.11.

A little more work is required to show the necessity of the denominator in Theorem 4.7. Let $d \in \mathbb{N}$ be sufficiently large (at least 127 , the dimension of the vector space of all polynomials in $X, X^{*}$ of degree at most 6). Suppose $M$ is cyclically equivalent to a polynomial $g$ that is positive semidefinite on $d \times d$ matrices. Without loss of generality, $g \in \operatorname{Sym} k\left\langle\underline{X}, \underline{X}^{*}\right\rangle$. Choose $g$ of the smallest possible degree. If this degree is greater than 6 , then the highest homogeneous component $g^{(\infty)}$ of $g$ is positive semidefinite on $d \times d$ matrices and at the same 
time $g^{(\infty)} \stackrel{\text { cyc }}{\sim} 0$. Hence $\operatorname{tr}\left(g^{(\infty)}\right)=0$ on $d \times d$ matrices, implying that $g^{(\infty)}$ is a polynomial identity. Then

$$
M \stackrel{\text { cyc }}{\sim}\left(g-g^{(\infty)}\right),
$$

with $g-g^{(\infty)}$ positive semidefinite and of degree smaller than $g$. This contradicts the minimality of $g$, $\operatorname{so} \operatorname{deg}(g) \leq 6$.

Now $g$ is positive semidefinite on $d \times d$ matrices for some $d \geq 127$ and is thus a sum of Hermitian squares by Helton's sum of squares theorem [2002]. But $M$ is not cyclically equivalent to a sum of Hermitian squares by the first part of this example.

\section{Acknowledgments}

The author thanks Matej Brešar for enlightening conversations and insightful comments.

\section{References}

[Amitsur and Rowen 1994] S. A. Amitsur and L. H. Rowen, "Elements of reduced trace 0", Israel J. Math. 87:1-3 (1994), 161-179. MR 95h:16019 Zbl 0852.16012

[Brešar and Klep 2009] M. Brešar and I. Klep, "Values of noncommutative polynomials, Lie skewideals and tracial Nullstellensätze", Math. Res. Lett. 16:4 (2009), 605-626. MR 2010m:16061 Zbl 1189.16021

[Cafuta et al. 2010] K. Cafuta, I. Klep, and J. Povh, "NCSOStools: a computer algebra system for symbolic and numerical computation with noncommutative polynomials", 2010, available at http:// www.optimization-online.org/DB_HTML/2010/05/2635.html. To appear in Optim. Methods Softw. Software available at http://ncsostools.fis.unm.si/.

[Collins and Dykema 2008] B. Collins and K. Dykema, "A linearization of Connes' embedding problem”, New York J. Math. 14 (2008), 617-641. MR 2010a:46141 Zbl 1162.46032

[Connes 1976] A. Connes, "Classification of injective factors: Cases $\mathrm{II}_{1}, \mathrm{II}_{\infty}, \mathrm{III}_{\lambda}, \lambda \neq 1$ ", Ann. Math. (2) 104:1 (1976), 73-115. MR 56 \#12908 Zbl 0343.46042

[Dieudonné 1952] J. Dieudonné, “On the structure of unitary groups", Trans. Amer. Math. Soc. 72:3 (1952), 367-385. MR 14,134c Zbl 0046.25301

[Helton 2002] J. W. Helton, “Positive' noncommutative polynomials are sums of squares", Ann. Math. (2) 156:2 (2002), 675-694. MR 2003k:12002 Zbl 1033.12001

[Helton et al. 2010] J. W. H. Helton, M. C. de Oliveira, R. L. Miller, and M. Stankus, "NCAlgebra: a Mathematica package for doing non-commuting algebra", January 2010, available at http:// www.math.ucsd.edu/ ncalg.

[Klep and Schweighofer 2008] I. Klep and M. Schweighofer, "Connes' embedding conjecture and sums of Hermitian squares", Adv. Math. 217:4 (2008), 1816-1837. MR 2009g:46109 Zbl 1184. 46055

[Klep and Unger 2010] I. Klep and T. Unger, "The Procesi-Schacher conjecture and Hilbert's 17th problem for algebras with involution", J. Algebra 324:2 (2010), 256-268. MR 2651356 Zbl 05768597 
[Knus et al. 1998] M.-A. Knus, A. S. Merkurjev, M. Rost, and J.-P. Tignol, The book of involutions, Amer. Math. Soc. Colloquium Publ. 44, AMS, Providence, RI, 1998. MR 2000a:16031 Zbl 0955.16001

[McCullough 2001] S. McCullough, "Factorization of operator-valued polynomials in several noncommuting variables", Linear Algebra Appl. 326 (2001), 193-203. MR 1815959 Zbl 0980.47024

[McCullough and Putinar 2005] S. McCullough and M. Putinar, "Noncommutative sums of squares", Pacific J. Math. 218:1 (2005), 167-171. MR 2007j:47026 Zbl 1177.47020

[Netzer and Thom 2010] T. Netzer and A. Thom, "Tracial algebras and an embedding theorem", $J$. Funct. Anal. 259:11 (2010), 2939-2960. MR 2719281 Zbl 05809715

[Procesi 1973] C. Procesi, Rings with polynomial identities, Pure and Applied Mathematics 17, Marcel Dekker, New York, 1973. MR 51 \#3214 Zbl 0262.16018

[Procesi 1976] C. Procesi, "The invariant theory of $n \times n$ matrices", Adv. Math. 19:3 (1976), 306381. MR 54 \#7512 Zbl 0331.15021

[Procesi and Schacher 1976] C. Procesi and M. Schacher, "A non-commutative real Nullstellensatz and Hilbert's 17th problem", Ann. Math. (2) 104:3 (1976), 395-406. MR 55 \#5599 Zbl 0347.16010

[Rowen 1980] L. H. Rowen, Polynomial identities in ring theory, Pure and Applied Mathematics 84, Academic Press, New York, 1980. MR 82a:16021 Zbl 0461.16001

[Saltman 1999] D. J. Saltman, Lectures on division algebras, CBMS Regional Conference Series in Mathematics 94, AMS, Providence, RI, 1999. MR 2000f:16023 Zbl 0934.16013

Received December 31, 2009.

IGOR KLEP

UNIVERZA V LJUBLJANI

FAKULTETA ZA MATEMATIKO IN FIZIKO

JADRANSKA 21

SI-1111 LJUBLJANA

SLOVENIA

and

UNIVERZA V MARIBORU

FAKULTETA ZA NARAVOSLOVJE IN MATEMATIKO

KOROŠKA 160

SI-2000 MARIBOR

SLOVENIA

igor.klep@fmf.uni-lj.si 


\title{
PACIFIC JOURNAL OF MATHEMATICS
}

\author{
http://www.pjmath.org \\ Founded in 1951 by
}

E. F. Beckenbach (1906-1982) and F. Wolf (1904-1989)

\section{EDITORS}

V. S. Varadarajan (Managing Editor)

Department of Mathematics

University of California

Los Angeles, CA 90095-1555

pacific@math.ucla.edu

Vyjayanthi Chari

Department of Mathematics

University of California

Riverside, CA 92521-0135

chari@math.ucr.edu

\section{Robert Finn}

Department of Mathematics Stanford University

Stanford, CA 94305-2125

finn@math.stanford.edu

Kefeng Liu

Department of Mathematics

University of California

Los Angeles, CA 90095-1555

liu@math.ucla.edu
Darren Long

Department of Mathematics

University of California

Santa Barbara, CA 93106-3080

long@math.ucsb.edu

Jiang-Hua Lu

Department of Mathematics

The University of Hong Kong

Pokfulam Rd., Hong Kong jhlu@maths.hku.hk

Alexander Merkurjev

Department of Mathematics University of California

Los Angeles, CA 90095-1555 merkurev@math.ucla.edu
Sorin Popa

Department of Mathematics

University of California

Los Angeles, CA 90095-1555

popa@math.ucla.edu

Jie Qing

Department of Mathematics

University of California

Santa Cruz, CA 95064

qing@ cats.ucsc.edu

Jonathan Rogawski

Department of Mathematics

University of California

Los Angeles, CA 90095-1555

jonr@math.ucla.edu

\section{PRODUCTION}

pacific@math.berkeley.edu

Silvio Levy, Scientific Editor Matthew Cargo, Senior Production Editor

ACADEMIA SINICA, TAIPEI

CALIFORNIA INST. OF TECHNOLOGY

INST. DE MATEMÁTICA PURA E APLICADA

KEIO UNIVERSITY

MATH. SCIENCES RESEARCH INSTITUTE

NEW MEXICO STATE UNIV.

OREGON STATE UNIV.

\section{SUPPORTING INSTITUTIONS}

STANFORD UNIVERSITY
UNIV. OF BRITISH COLUMBIA
UNIV. OF CALIFORNIA, BERKELEY
UNIV. OF CALIFORNIA, DAVIS
UNIV. OF CALIFORNIA, LOS ANGELES
UNIV. OF CALIFORNIA, RIVERSIDE
UNIV. OF CALIFORNIA, SAN DIEGO
UNIV. OF CALIF., SANTA BARBARA

UNIV. OF CALIF., SANTA CRUZ

UNIV. OF MONTANA

UNIV. OF OREGON

UNIV. OF SOUTHERN CALIFORNIA

UNIV. OF UTAH

UNIV. OF WASHINGTON

WASHINGTON STATE UNIVERSITY

These supporting institutions contribute to the cost of publication of this Journal, but they are not owners or publishers and have no responsibility for its contents or policies.

See inside back cover or www.pjmath.org for submission instructions.

The subscription price for 2011 is US \$420/year for the electronic version, and \$485/year for print and electronic.

Subscriptions, requests for back issues from the last three years and changes of subscribers address should be sent to Pacific Journal of Mathematics, P.O. Box 4163, Berkeley, CA 94704-0163, U.S.A. Prior back issues are obtainable from Periodicals Service Company, 11 Main Street, Germantown, NY 12526-5635. The Pacific Journal of Mathematics is indexed by Mathematical Reviews, Zentralblatt MATH, PASCAL CNRS Index, Referativnyi Zhurnal, Current Mathematical Publications and the Science Citation Index.

The Pacific Journal of Mathematics (ISSN 0030-8730) at the University of California, c/o Department of Mathematics, 969 Evans Hall, Berkeley, CA 94720-3840, is published monthly except July and August. Periodical rate postage paid at Berkeley, CA 94704, and additional mailing offices. POSTMASTER: send address changes to Pacific Journal of Mathematics, P.O. Box 4163, Berkeley, CA 94704-0163.

PJM peer review and production are managed by EditFLOW ${ }^{\mathrm{TM}}$ from Mathematical Sciences Publishers.

PUBLISHED BY PACIFIC JOURNAL OF MATHEMATICS

at the University of California, Berkeley 94720-3840

A NON-PROFIT CORPORATION

Typeset in IATEX

Copyright $(2011$ by Pacific Journal of Mathematics 


\section{PACIFIC JOURNAL OF MATHEMATICS}

Volume $250 \quad$ No. $2 \quad$ April 2011

Realizing profinite reduced special groups

VinCENT Astier and Hugo Mariano

On fibered commensurability

DANNy CALEGARI, Hongbin SUN and Shicheng WANG

On an overdetermined elliptic problem

LAURENT HaUswirTh, FrÉdÉRIC HÉLEIN and FranK PACARD

Minimal sets of a recurrent discrete flow

HATTAB HAWETE

Trace-positive polynomials

IGOR KLEP

Remarks on the product of harmonic forms

LiviU ORnea and Mihaela Pilca

Steinberg representation of GSp(4): Bessel models and integral representation of $L$-functions

Ameya Pitale

An integral expression of the first nontrivial one-cocycle of the space of long knots in $\mathbb{R}^{3}$

KEIICHI SAKAI

Burghelea-Haller analytic torsion for twisted de Rham complexes

GUANGXIANG SU

$K(n)$-localization of the $K(n+1)$-local $E_{n+1}$-Adams spectral sequences

TAKESHI TORII

Thompson's group is distorted in the Thompson-Stein groups

Claire Wladis

Parabolic meromorphic functions 